\title{
Sensitivity with Respect to the Path Parameters and Nonlinear Stiffness of Vibration Transfer Path Systems
}

\author{
Yimin Zhang and Xianzhen Huang \\ School of Mechanical Engineering and Automation, Northeastern University, Shenyang 110004, China \\ Correspondence should be addressed to Yimin Zhang, neu831109@yahoo.com.cn
}

Received 17 December 2009; Accepted 6 April 2010

Academic Editor: Carlo Cattani

Copyright (C) 2010 Y. Zhang and X. Huang. This is an open access article distributed under the Creative Commons Attribution License, which permits unrestricted use, distribution, and reproduction in any medium, provided the original work is properly cited.

Generally speaking, a vibration system consists of three parts: vibration resource, vibration transfer path, and vibration receiver. Based on the dynamic sensitivity technique, this paper proposes a method for evaluating the contribution of each vibration transfer path to the dynamic response of the vibration receiver. Nonlinear stiffness is an important factor in causing the nonlinearity of vibration systems. Taking sensitivity as the evaluation criteria, we present an effective approach for estimating the influence of nonlinear stiffness in vibration transfer paths on the dynamic response of the vibration receiver. Using the proposed method, the sensitivity of the vibration system with multiple and/or multidimensional transfer paths could be determined in the time domain.

\section{Introduction}

The level of vibration and noise relates to the quality of mechanical products and equipments. That is to say mechanical products with proper vibration and noise characteristics are prone to be favored by customers and are prone to make more profits for the enterprise. Therefore, the study of the transfer of vibration and noise in vibration systems with multiple and/or multi-dimensional transfer paths is of significant value. As well known, the technology of vibration and noise control plays an important role both in the improvement of the comprehensive property and technical index of mechanical products and in the advancement of the scientific and technical grade of mechanical equipments. The conventional conception of vibration and noise control in practical engineering projects is to cut off the transfer path of vibration and noise so as to reduce vibration and noise disturbance. However, in practical applications, it is out of the question to completely cut off the vibration transfer path. And an engineer can only try to minimize the energy transfer in vibration 


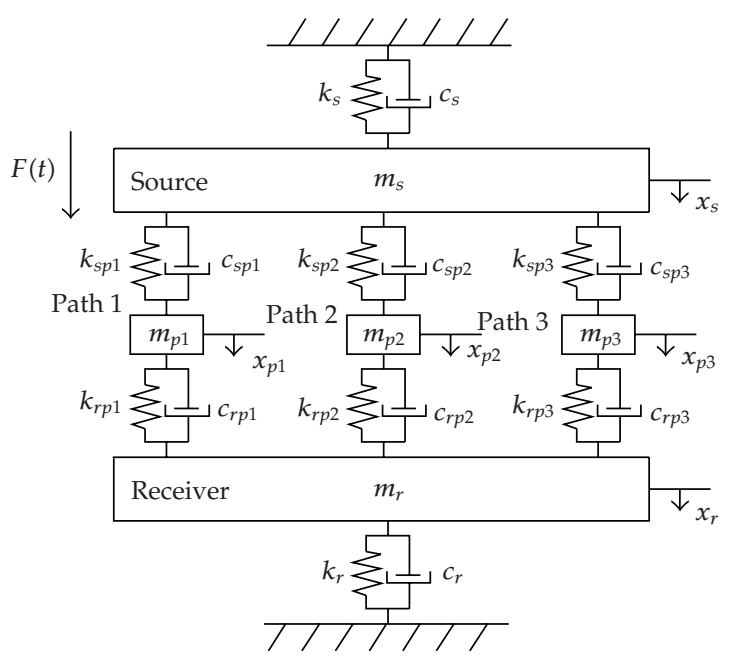

Figure 1: The model of 5-DOF vibration transfer path systems.

transfer paths while the vibration transfer paths in vibration systems are precisely identified. Therefore, the researches on the interaction of the vibration source, vibration transfer path, and vibration receiver are mainly focused on energy transfer techniques and physical experiments. The methods based on energy (or power) transfer and dynamic tests have been widely used to investigate the dynamic characteristics of vibration transfer path systems [15].

When conducting a dynamic design or modification, a designer always needs to know which parameters have more significant effects on the dynamic performance of the system. Therefore, dynamic sensitivity analysis has turned to be a powerful and multipurpose design tool in optimum structural design, structural parameter identification, system dynamic control, and so forth. Generally speaking, vibration tests are needed in dynamic design of a practical engineering project. Once the design scheme is changed, the whole process of the vibration test should be performed once again, which wastes great expenditures of resources, both human and material. In this case, a designer relies largely upon his experiences and past works, which is blind to some extent. Therefore, we need to study the sensitivity of vibration systems with respect to path parameters. In this way, the performance variation of the dynamic system following the change of path parameters can be figured out.

On the basis of the basic conception of vibration theories, this paper presents a method to quantify the contribution of each vibration transfer path to the dynamic response of the vibration transfer path system by employing the dynamic sensitivity as an evaluation index. Especially, a method for sensitivity analysis of nonlinear vibration transfer path systems with respect to different kinds of non-linear stiffness is also explored in the time domain. In view of the above studies, the designer can figure out which kind of non-liner stiffness has more significant influence on the dynamic response of the vibration transfer path system. Then precautionary measures can be taken to reduce the energy transfer of vibration and noise by modifying the structural parameter and topology or by changing components in the significant transfer paths. 


\section{Vibration Transfer Path Systems}

Generally speaking, every vibration engineering problem can be rationalized into three parts: vibration source (i.e., what is the dynamic loading), vibration path (i.e., what is the structural mass, stiffness, and damping), and vibration response and its effects on the "receiver" (typically, the receiver is humans, occupying the structure but could also be vibration sensitive equipment). As shown in Figure 1, a five-degree-of-freedom (5-DOF) vibration transfer path system with non-linear stiffness is subjected to a dynamic excitation. Newton's law can be applied to obtain the vibration differential equations of the 5-DOF vibration transfer path system as follows:

$$
\mathbf{M} \ddot{\mathbf{x}}+\mathbf{C} \dot{\mathbf{x}}+\mathbf{K}(\mathbf{x}) \mathbf{x}=\mathbf{F}(t),
$$

where

$$
\begin{aligned}
& \mathbf{M}=\operatorname{diag}\left[\begin{array}{lllll}
m_{s} & m_{p 1} & m_{p 2} & m_{p 3} & m_{r}
\end{array}\right], \\
& \mathbf{C}=\left[\begin{array}{ccccc}
c_{s}+c_{s p 1}+c_{s p 2}+c_{s p 3} & -c_{s p 1} & -c_{s p 2} & -c_{s p 3} & 0 \\
-c_{s p 1} & c_{s p 1}+c_{r p 1} & 0 & 0 & -c_{r p 1} \\
-c_{s p 2} & 0 & c_{s p 2}+c_{r p 2} & 0 & -c_{r p 2} \\
-c_{s p 3} & 0 & 0 & c_{s p 3}+c_{r p 3} & -c_{r p 3} \\
0 & -c_{r p 1} & -c_{r p 2} & -c_{r p 3} & c_{r}+c_{r p 1}+c_{r p 2}+c_{r p 3}
\end{array}\right], \\
& \mathbf{K}=\left[\begin{array}{ccccc}
k_{s}+k_{s p 1}+k_{s p 2}+k_{s p 3} & -k_{s p 1} & -k_{s p 2} & -k_{s p 3} & 0 \\
-k_{s p 1} & k_{s p 1}+k_{r p 1} & 0 & 0 & -k_{r p 1} \\
-k_{s p 2} & 0 & k_{s p 2}+k_{r p 2} & 0 & -k_{r p 2} \\
-k_{s p 3} & 0 & 0 & k_{s p 3}+k_{r p 3} & -k_{r p 3} \\
0 & -k_{r p 1} & -k_{r p 2} & -k_{r p 3} & k_{r}+k_{r p 1}+k_{r p 2}+k_{r p 3}
\end{array}\right] \text {, } \\
& \mathbf{F}(t)=\left\{F_{0} \sin (\omega t) 0000\right\}^{T}, \quad \mathbf{x}(t)=\left\{x_{\mathrm{s}} x_{p 1} x_{p 2} x_{p 3} x_{r}\right\}^{T} .
\end{aligned}
$$

In this paper, $k_{s p 1}$ is supposed to be non-linear stiffness. In general, there are two kinds of nonlinear elastic forces, which are called the material non-linear force and the piecewise linear force. $k_{s p 1}^{e(i)}(i=1,2)$ is used to denote the equivalent linear stiffness of the above mentioned two kinds of non-linear stiffness. In engineering practice, the non-linear stiffness is usually approximately described by equivalent linear stiffness as

$$
\mathbf{K}^{e}=\frac{\partial \mathbf{F}(\mathbf{x})}{\partial \mathbf{x}^{T}}=\frac{\partial[\mathbf{K}(\mathbf{x}) \mathbf{x}]}{\partial \mathbf{x}^{T}}=\mathbf{K}(\mathbf{x})+\frac{\partial \mathbf{K}(\mathbf{x})}{\partial \mathbf{x}^{T}}\left(\mathbf{I}_{n} \otimes \mathbf{x}\right),
$$


where $\mathbf{F}(\mathbf{x})=\mathbf{K}(\mathbf{x}) \mathbf{x}$ denotes the non-linear elastic force, $\mathbf{I}_{n}$ is a identity matrix with $n \times n$ dimensions, and the symbol $\otimes$ represents Kronecker product, which can be defined as

$$
A_{p \times q} \otimes B_{s \times t}=\left[\begin{array}{cccc}
a_{11} B & a_{12} B & \cdots & a_{1 q} B \\
a_{21} B & a_{22} B & \cdots & a_{2 q} B \\
\vdots & \vdots & \ddots & \vdots \\
a_{p 1} B & a_{p 2} B & \cdots & a_{p q} B
\end{array}\right]_{p s \times q t}
$$

\section{Nonlinear Stiffness in Vibration Transfer Path Systems}

In most cases, the elastic force is non-linear. When dealing with a specific problem, based on experiments, the non-linear elastic force can always be expressed as a function of motion parameters after some simplifications. This approach is often an important step in dealing with vibration engineering problems with non-linear stiffness.

\subsection{Material Non-Linear Stiffness}

In actual engineering materials, the stress-strain relation is nonlinear and follows Hook's law only up to a certain deformation (displacement of one end with respect to the other). Beyond the deformation, the stress exceeds the yield point of the material and the stressstrain relation becomes nonlinear. This kind of non-linear stiffness is usually called material non-linear stiffness, which can be roughly divided into two types. (1) If the slope of the forcedeflection curve increases with the increase of the deformation, the spring element is said to be hardening or hard. (2) If the slope of the force-deflection curve decreases with the increase of the deformation, the spring element is softening or soft. In many practical applications, the non-linear elastic force can be approximately denoted as

$$
F_{s p 1}^{(1)}(\mathbf{x})=k_{s p 1}^{1(1)}\left(x_{p 1}-x_{s}\right) \pm k_{s p 1}^{2(1)}\left(x_{p 1}-x_{s}\right)^{3}
$$

where $k_{s p 1}^{1(1)}>0, k_{s p 1}^{2(1)}>0$ and $x_{p 1}-x_{s}$ is the displacement of the spring from its free length, $k_{s p 1}^{1(1)}\left(x_{p 1}-x_{s}\right)$ denotes the linear elastic force, $k_{s p 1}^{2(1)}\left(x_{p 1}-x_{s}\right)^{3}$ denotes the non-linear elastic force, and the non-linear elastic force is often much smaller than the linear elastic force. Therefore the non-linear elastic force can be regarded as a correction term. Sign " \pm " indicates the character of stiffness, which is " + " or " - " depending on whether the character of the stiffness is hard or soft. Consequently, the coefficient of the equivalent linear stiffness, $k_{s p 1}^{e(1)}$, of material non-linear stiffness can be represented as

$$
k_{s p 1}^{e(1)}=k_{s p 1}^{1(1)} \pm 3 k_{s p 1}^{2(1)}\left(x_{p 1}-x_{s}\right)^{2} .
$$

As shown in (3.2), a nonlinear spring element does not have a single stiffness value because its slope is variable. For a hard spring, its slop and thus its stiffness increase with deflection. The stiffness of a soft spring decreases with deflection. 


\subsection{Piecewise Linear Stiffness}

Sometimes a nonlinear model is unavoidable. This is the case when a system is designed to utilize two or more spring elements to achieve a spring constant that varies with the applied load. Even if each spring element is linear, the combined system will be nonlinear. An example of such a system is the vehicle suspension with a main spring and an auxiliary spring. Furthermore, some vibrating machineries, such as the vibrating screen and the oscillating conveyer, use the spring set to get the desired dynamic characteristics. As for this kind of non-linear elastic model, under certain circumstances, two or more groups of springs work together in the form of parallel or series to increase or decrease the stiffness of the system. From statics, we know that the non-linear restoring force of the piecewise linear stiffness vibration system can be represented as

$$
F_{s p 1}^{(2)}(\mathbf{x})= \begin{cases}k_{s p 1}^{2(2)}\left(x_{p 1}-x_{s}\right)-\left(k_{s p 1}^{1(2)}-k_{s p 1}^{2(2)}\right)\left(x_{p 1}-x_{s}\right)_{0^{\prime}} & -\left(x_{p 1}-x_{s}\right)_{0} \geq\left(x_{p 1}-x_{s}\right), \\ k_{s p 1}^{1(2)}\left(x_{p 1}-x_{s}\right), & -\left(x_{p 1}-x_{s}\right)_{0} \leq\left(x_{p 1}-x_{s}\right) \leq\left(x_{p 1}-x_{s}\right)_{0^{\prime}} \\ k_{s p 1}^{2(2)}\left(x_{p 1}-x_{s}\right)+\left(k_{s p 1}^{1(2)}-k_{s p 1}^{2(2)}\right)\left(x_{p 1}-x_{s}\right)_{0^{\prime}} & \left(x_{p 1}-x_{s}\right)_{0} \leq\left(x_{p 1}-x_{s}\right) .\end{cases}
$$

Therefore, for the non-linear stiffness of piecewise linearity, the equivalent stiffness $k_{s p 1}^{e(2)}$ of $k_{s p 1}$ is

$$
k_{s p 1}^{e(2)}= \begin{cases}k_{s p 1}^{1(2)}, & -\left(x_{p 1}-x_{s}\right)_{0} \leq\left(x_{p 1}-x_{s}\right) \leq\left(x_{p 1}-x_{s}\right)_{0} . \\ k_{s p 1}^{2(2)}, & \text { otherwise. }\end{cases}
$$

To investigate the influences of different kinds of non-linear stiffness on the dynamic response of the vibration transfer path system, we present a method for sensitivity analysis of the dynamic response of the vibration receiver with respect to the equivalent linear stiffness coefficients $\left(k_{s p 1}^{e(1)}\right.$ and $\left.k_{s p 1}^{e(2)}\right)$ of material non-linear stiffness and piecewise linear stiffness. In this way, which kind of nonlinear stiffness has more significant influence on the dynamic responses of vibration transfer path systems can be accurately judged.

\section{Sensitivity with Respect to Path Parameters and Non-Linear Stiffness}

Sensitivity analysis plays an important role in optimization design and dynamic modification. Based on design sensitivity results, an engineer can decide on the direction and amount of design change needed to improve the performance measures. In addition, design sensitivity information can provide answers to "what if" questions by predicting performance measure perturbations when the perturbations of design variables are provided. The dynamic sensitivity analysis provides a theoretical basis for identifying the parameter contribution of each transfer path to the dynamic response of vibration transfer path systems. 
Based on Kronecker algebra and matrix calculus, we can obtain general sensitivity equations through partial differential vector calculus of (2.1):

$$
\mathbf{M} \frac{D \ddot{\mathbf{x}}}{D \mathbf{V}^{T}}+\mathbf{C} \frac{D \dot{\mathbf{x}}}{D \mathbf{V}^{T}}+\mathbf{K} \frac{D \mathbf{x}}{D \mathbf{V}^{T}}=\frac{\mathbf{F}(\mathbf{t})}{\partial \mathbf{V}^{T}}-\frac{\partial \mathbf{M}}{\partial \mathbf{V}^{T}}\left(\mathbf{I}_{s} \otimes \ddot{\mathbf{x}}\right)-\frac{\partial \mathbf{C}}{\partial \mathbf{V}^{T}}\left(\mathbf{I}_{s} \otimes \dot{\mathbf{x}}\right)-\frac{\partial \mathbf{K}}{\partial \mathbf{V}^{T}}\left(\mathbf{I}_{s} \otimes \mathbf{x}\right),
$$

where $\mathbf{I}_{s}$ is a identity matrix with $s \times s$ dimensions, signal " $\otimes$ " is the Kronecker

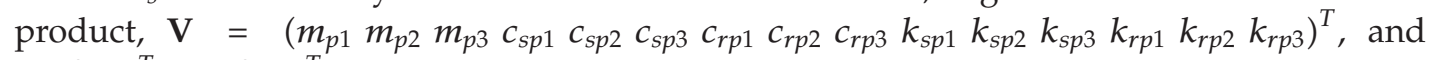
$D \mathbf{x} / D \mathbf{V}^{T}, D \dot{\mathbf{x}} / D \mathbf{V}^{T}$,

$D \ddot{\mathbf{x}} / D \mathbf{V}^{T}$ are Jacobian matrices, namely, the parameter sensitivity matrices. And the parameter sensitivity matrices can be obtained by substituting the solutions of $(2.1), \mathbf{x}, \dot{\mathbf{x}}, \ddot{\mathbf{x}}$ into (4.1). Then the sensitivity of dynamic response of the vibration transfer path system with respect to each path parameter can be solved as follows:

$$
\begin{aligned}
\frac{D \mathbf{x}}{D m_{p}} & =\frac{\partial \mathbf{x}}{\partial m_{p 1}}+\frac{\partial \mathbf{x}}{\partial m_{p 2}}+\frac{\partial \mathbf{x}}{\partial m_{p 3}}, \\
\frac{D \mathbf{x}}{D c_{s p}} & =\frac{\partial \mathbf{x}}{\partial c_{s p 1}}+\frac{\partial \mathbf{x}}{\partial c_{s p 2}}+\frac{\partial \mathbf{x}}{\partial c_{s p 3}}, \\
\frac{D \mathbf{x}}{D c_{r p}} & =\frac{\partial \mathbf{x}}{\partial c_{r p 1}}+\frac{\partial \mathbf{x}}{\partial c_{r p 2}}+\frac{\partial \mathbf{x}}{\partial c_{r p 3}}, \\
\frac{D \mathbf{x}}{D k_{s p}} & =\frac{\partial \mathbf{x}}{\partial k_{s p 1}}+\frac{\partial \mathbf{x}}{\partial k_{s p 2}}+\frac{\partial \mathbf{x}}{\partial k_{s p 3}}, \\
\frac{D \mathbf{x}}{D k_{r p}} & =\frac{\partial \mathbf{x}}{\partial k_{r p 1}}+\frac{\partial \mathbf{x}}{\partial k_{r p 2}}+\frac{\partial \mathbf{x}}{\partial k_{r p 3}},
\end{aligned}
$$

where $D \mathbf{x} / D m_{p}, D \mathbf{x} / D c_{s p}, D \mathbf{x} / D c_{r p}, D \mathbf{x} / D k_{s p}$, and $D \mathbf{x} / D k_{r p}$ are the sensitivity of the dynamic response of the vibration receiver with respect to the mass, damping and stiffness of the vibration transfer path system, respectively. These sensitivity matrices can be used to judge the contribution of each path parameter to the dynamic response of the vibration receiver. The sign of sensitivity can be positive or negative. When a sensitivity coefficient is positive, an increase in the model parameter leads to an increase in the corresponding state variable, and when it is negative, the opposite is true. Moreover, the larger the absolute value of sensitivity is, the more significant is the factor for the response of the vibration transfer path system.

Sensitivity analysis of a vibration transfer path system with respect to non-linear path stiffness provides a theoretical basis for identifying the parameter contribution of each kind of non-linear stiffness to the dynamic response of the vibration receiver. Through the differential of (2.1) with respect to $k_{s p 1}^{e(i)}$, we get the following sensitivity function:

$$
\mathbf{M} \frac{\mathrm{d} \ddot{\mathbf{x}}}{\mathrm{d} k_{s p 1}^{e(i)}}+\mathbf{C} \frac{\mathrm{d} \dot{\mathbf{x}}}{\mathrm{d} k_{s p 1}^{e(i)}}+\mathbf{K} \frac{\mathrm{d} \mathbf{x}}{\mathrm{d} k_{s p 1}^{e(i)}}=-\frac{\mathrm{d} \mathbf{K}}{\mathrm{d} k_{s p 1}^{e(i)}} \mathbf{x} \quad(i=1,2)
$$




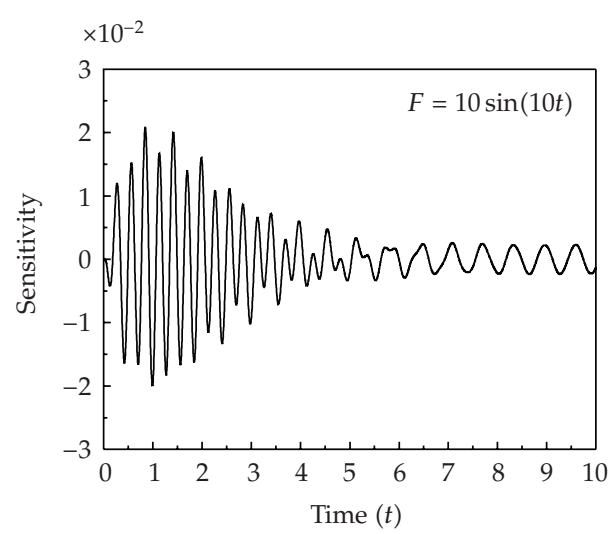

(a)

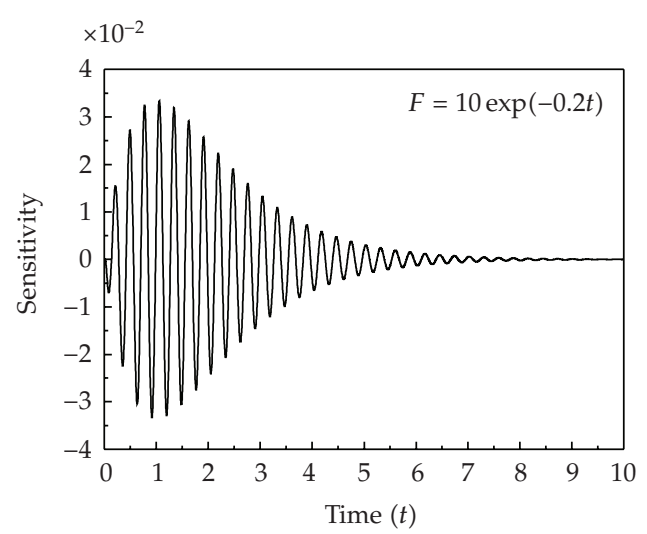

(b)

Figure 2: Sensitivity with respect to mass.

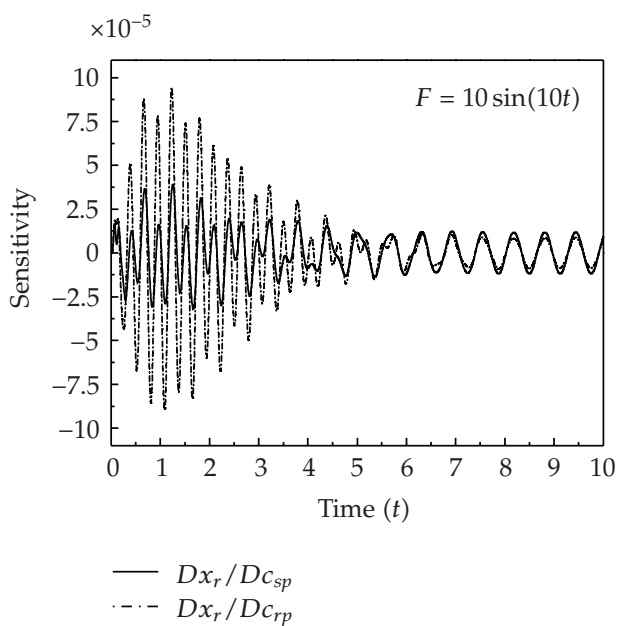

(a)

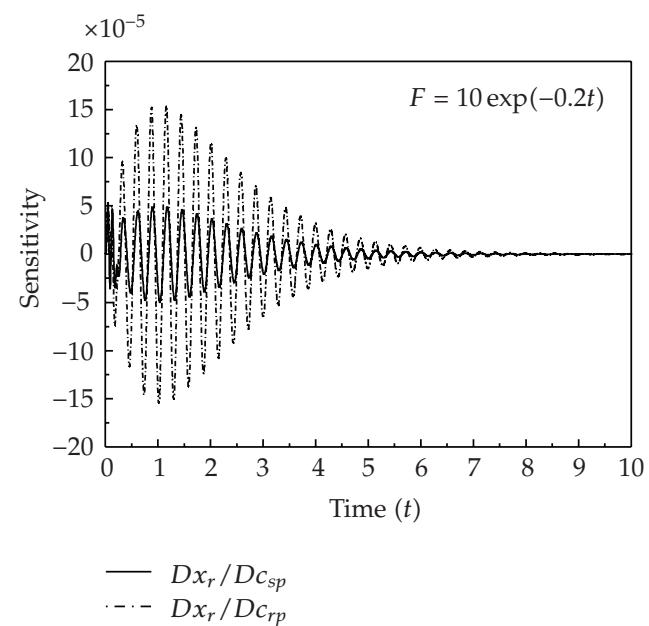

(b)

Figure 3: Sensitivity with respect to damping.

where $\mathrm{d} \mathbf{x} / \mathrm{d} k_{s p 1}^{e(i)}, \mathrm{d} \dot{\mathbf{x}} / \mathrm{d} k_{s p 1}^{e(i)}$, and $\mathrm{d} \ddot{\mathbf{x}} / \mathrm{d} k_{s p 1}^{e(i)}$ are sensitivity matrices of the dynamic response of the vibration receiver with respect to the coefficient of non-linear path stiffness. In order to identify the contribution of different kinds of non-linear stiffness in transfer paths, we present a sensitivity analysis model by which the sensitivity of the vibration transfer path system with respect to the coefficients of different kinds of non-linear stiffness, $\mathbf{V}=\left(k_{s p 1}^{2(1)} k_{s p 1}^{2(2)}\right)^{T}$, can be obtained. Obviously, we can get the dynamic response of the vibration transfer path system, $\mathbf{x}, \dot{\mathbf{x}}$, and $\ddot{\mathbf{x}}$, from (2.1). Then, substituting the results of (2.1) into (4.1) and (4.3), we can get the sensitivity of the vibration transfer path system with respect to linear equivalent stiffness coefficients $k_{s p 1}^{2(1)}$ and $k_{s p 1}^{2(2)}$ to evaluate the effects of the non-linear stiffness on the dynamic response of the vibration receiver. 


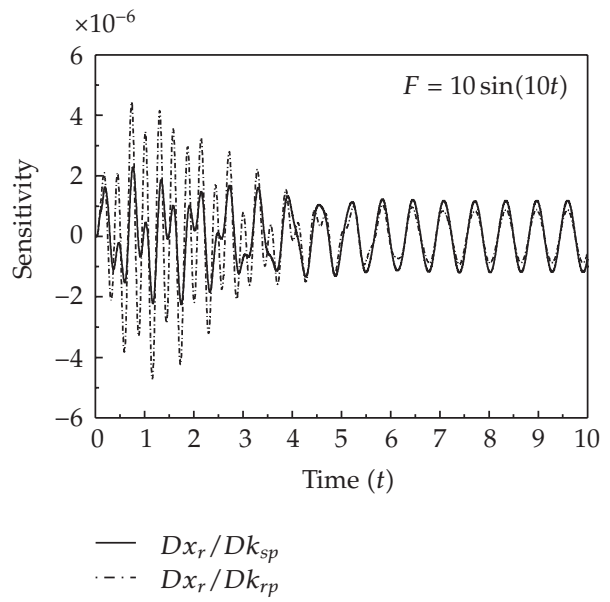

(a)

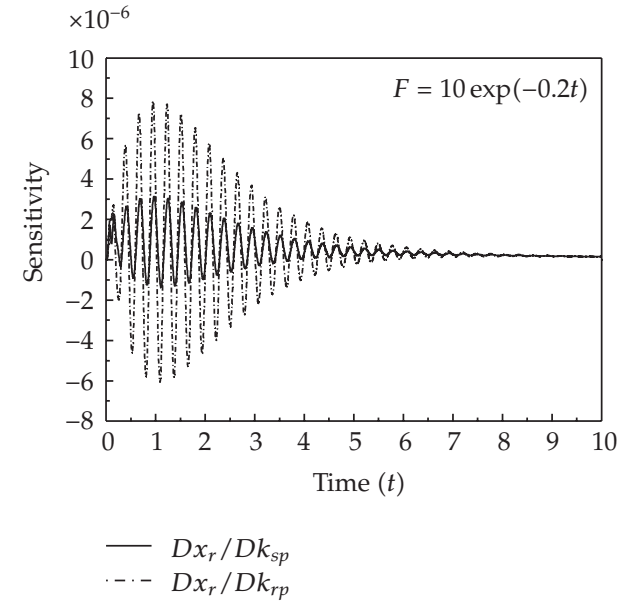

(b)

Figure 4: The curve of the sensitivity with respect to stiffness.

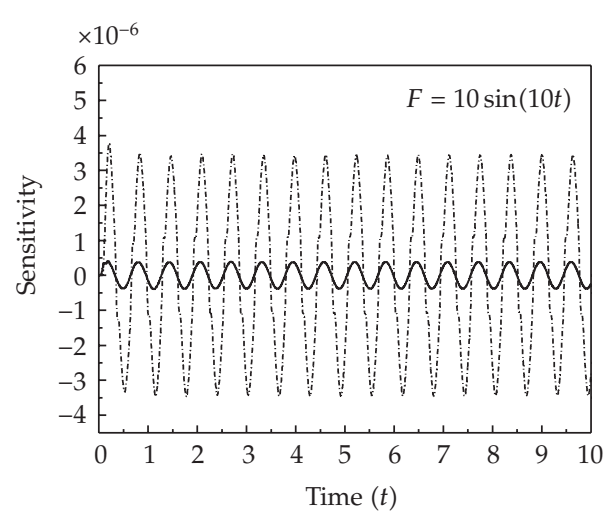

$-D x_{r} / D k_{s p 1}^{e(1)} \cdots D x_{r} / D k_{s p 1}^{e(2)}$

(a)

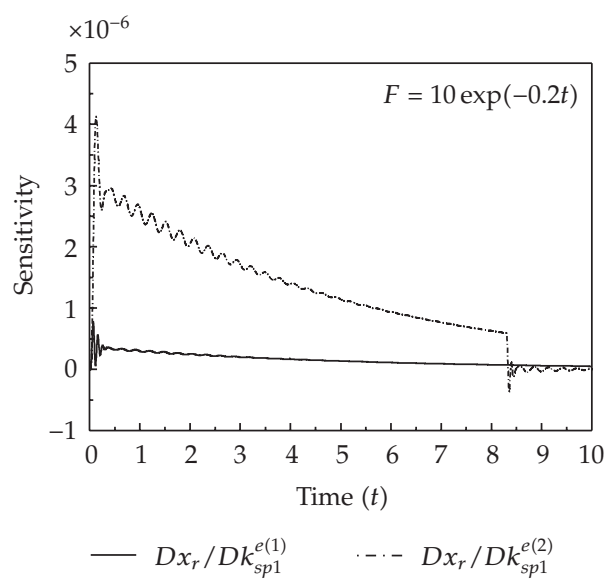

(b)

Figure 5: Sensitivity with respect to equivalent stiffness.

\section{Numerical Examples}

As the 5-DOF vibration transfer path system shown in Figure 1, the mass of the vibration resource is $m_{s}=0.5 \mathrm{~kg}$, the damping coefficient of the vibration resource system is $c_{s}=1 \mathrm{~N}$. $\mathrm{s} / \mathrm{m}$, and the stiffness coefficient of the vibration resource system is $k_{s}=500 \mathrm{~N} / \mathrm{m}$, the mass of the vibration receiver is $m_{r}=0.5 \mathrm{~kg}$, the damping coefficient of the vibration receiver system is $c_{r}=1.0 \mathrm{~N} \cdot \mathrm{s} / \mathrm{m}$, the stiffness of the vibration receiver system is $k_{r}=1000 \mathrm{~N} / \mathrm{m}$. In the three transfer paths, the masses and the coefficients of the damping and stiffness are $m_{p 1}=0.4 \mathrm{~kg}$, $m_{p 2}=0.5 \mathrm{~kg}, m_{p 3}=0.6 \mathrm{~kg}, c_{s p 1}=c_{r p 1}=6 \mathrm{~N} \cdot \mathrm{s} / \mathrm{m}, c_{s p 2}=c_{r p 2}=4 \mathrm{~N} \cdot \mathrm{s} / \mathrm{m}, c_{s p 3}=c_{r p 3}=8 \mathrm{~N} \cdot \mathrm{s} / \mathrm{m}$, $k_{s p 1}=k_{r p 1}=800 \mathrm{~N} / \mathrm{m}, k_{s p 2}=k_{r p 2}=600 \mathrm{~N} / \mathrm{m}$, and $k_{s p 3}=k_{r p 3}=400 \mathrm{~N} / \mathrm{m}$, respectively. Try to figure out the sensitivity of the vibration transfer path system with respect to each path parameter when subjected to dynamic excitation, $F=10 \sin (10 t)$ or $F=10 \exp (-0.2 t)$. 


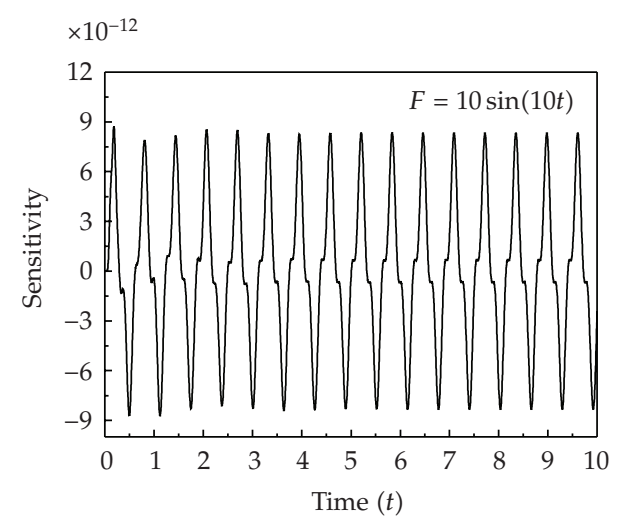

(a)

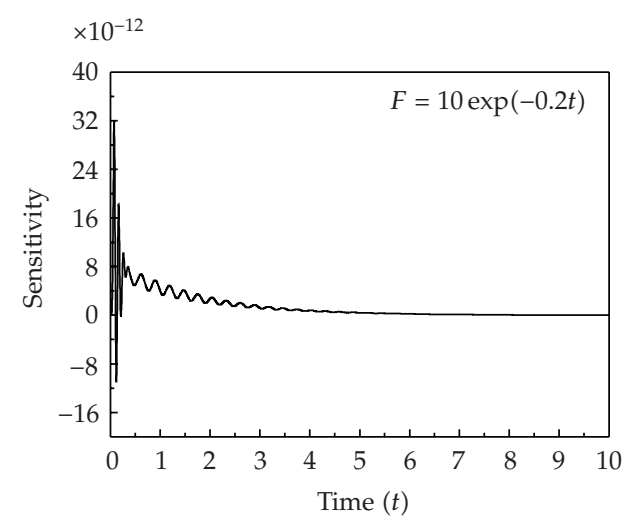

(b)

Figure 6: Sensitivity with respect to the material non-linear coefficient $k_{s p 1}^{2(2)}$.

The sensitivity of the vibration transfer path system with respect to the mass, damping and stiffness of the vibration transfer path system can be depicted in Figures 2-4. From the results of sensitivity analysis, we can draw the conclusion that under the same excitation, the mass has the strongest influence on the vibration transfer path system, followed by the damping, and the stiffness. Besides, the damping and stiffness near the vibration receiver have stronger effect on the dynamic response of the vibration receiver at the transient vibration stage. Therefore, while modifying structural parameters or replacing components, the mass, damping and stiffness of the 5-DOF vibration transfer path system need to be sequentially considered, so that the transfer of vibration and noise in vibration transfer path systems can be decreased efficiently.

In the above mentioned 5-DOF vibration transfer path system, $k_{s p 1}$ is non-linear stiffness, and the liner parts of the non-linear stiffness are $k_{s p 1}^{1(1)}=k_{s p 1}^{1(2)}=800 \mathrm{~N} / \mathrm{m}$. The turning point of the piecewise linear stiffness is $\left(x_{p 1}-x_{s}\right)_{0}=1.5 \times 10^{-3} \mathrm{~m}$, and $k_{s p 1}^{2(1)}=80 \mathrm{~N} / \mathrm{m}^{3}$. The nonlinear correction term of the non-linear material stiffness is $k_{s p 1}^{2(2)}=8 \mathrm{~N} / \mathrm{m}$. Try to figure out the sensitivity of the dynamic response of the 5-DOF vibration transfer path system with respect to each kind of non-linear stiffness.

(1) The sensitivity of the dynamic response of the vibration receiver in the 5-DOF vibration transfer path system with respect to the linear equivalent stiffness coefficient of the non-linear stiffness $k_{s p 1}^{e(i)}(i=1,2)$ is depicted in Figure 5:

From Figure 5, we can draw the conclusion that although the linear parts of nonlinear stiffness are equal, the sensitivity with respect to the linear equivalent stiffness of material nonlinear stiffness and piecewise linear stiffness is different. The reason is that the dependency relationship between the non-linear part of different kinds of non-linear stiffness and the dynamic response of the vibration transfer path system is different. In comparison, the sensitivity with respect to the equivalent linear stiffness of the piecewise linear stiffness is bigger than that of the material non-linear stiffness.

(2) The sensitivity of the dynamic response of the vibration receiver in the 5-DOF vibration transfer path system with respect to the coefficients of the non-linear part of the material non-linear stiffness and piecewise stiffness, $k_{s p 1}^{2(1)}$ and $k_{s p 1}^{2(2)}$, is depicted in Figures 6 and 7. 


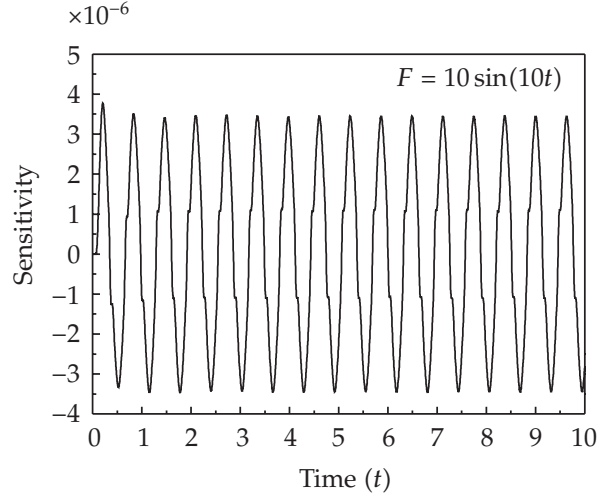

(a)

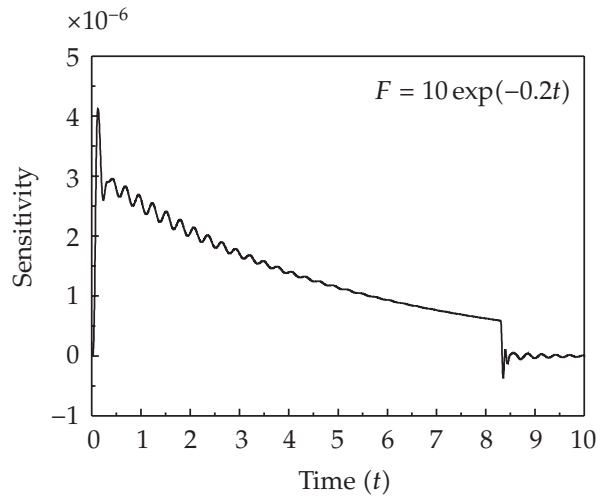

(b)

Figure 7: Sensitivity with respect to the piecewise linear coefficient $k_{s p 1}^{2(2)}$.

From the results shown in Figures 6 and 7, we can draw the conclusion that in the above mentioned 5-DOF vibration transfer path system, the non-linear part of the piecewise stiffness, $k_{s p 1}^{2(2)}$, is more sensitive to the dynamic responses of the vibration transfer path system than that of the material non-linear stiffness, $k_{s p 1}^{2(1)}$. However these two non-linear stiffness parameters have different units; the results drawn above can only be used as a reference to decrease the transfer of vibration or noise in the vibration transfer path system.

\section{Conclusion}

The effects of non-linear stiffness parameters on vibration transfer path systems are discussed in this paper. Based on the sensitivity technology, sensitivity scheduling of dynamic responses of vibration transfer path systems with respect to path parameters and non-linear stiffness is provided in the time-domain. The units of mass and the coefficients of different kinds of nonlinear stiffness are different; therefore the results drawn in this paper can only be used as a reference to decrease the transfer of vibration and noise in vibration transfer path systems. However, without a doubt, this paper provides an effective way to analyze the sensitivity of non-linear vibration transfer path systems with respect to path parameters and non-linear stiffness.

\section{Acknowledgments}

The authors would like to express their appreciation to Program for Changjiang Scholars and Innovative Research Team in university, Key National Science \& Technology Special Project on "High-Grade CNC Machine Tools and Basic Manufacturing Equipment" (2010ZX04014014), Chinese National Natural Science Foundation (50875039), and Fundamental Research Funds for the Central Universities (N090603002). Thanks to Professor R. Singh of The Ohio State University for providing the vibration path model (Figure 1) during the first author's visiting as a scholar. 


\section{References}

[1] L. Cremer and M. Heckle, Structure-Borne Sound: Structural Vibrations and Sound Radiation at Audio Frequencies, Springer, New York, NY, USA, 1973.

[2] R. H. Lyon and R. G. Dejong, Theory and Application of Statistical Energy Analysis, ButterworthHeinemann, Boston, Mass, USA, 1995.

[3] J. C. Wohlever and R. J. Bernhard, "Mechanical energy flow models of rods and beams," Journal of Sound and Vibration, vol. 153, no. 1, pp. 1-19, 1992.

[4] S. Kim and R. Singh, "Vibration transmission through an isolator modelled by continuous system theory," Journal of Sound and Vibration, vol. 248, no. 5, pp. 925-953, 2001.

[5] R. Singh and S. Kim, "Examination of multi-dimensional vibration isolation measures and their correlation to sound radiation over a broad frequency range," Journal of Sound and Vibration, vol. 262, no. 3, pp. 419-455, 2003. 


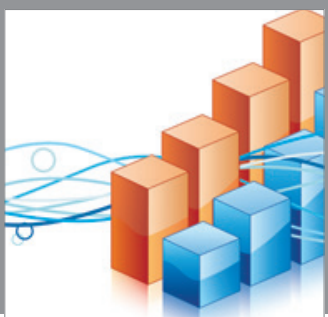

Advances in

Operations Research

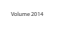

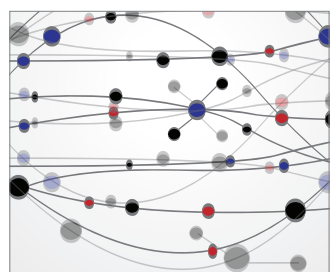

\section{The Scientific} World Journal
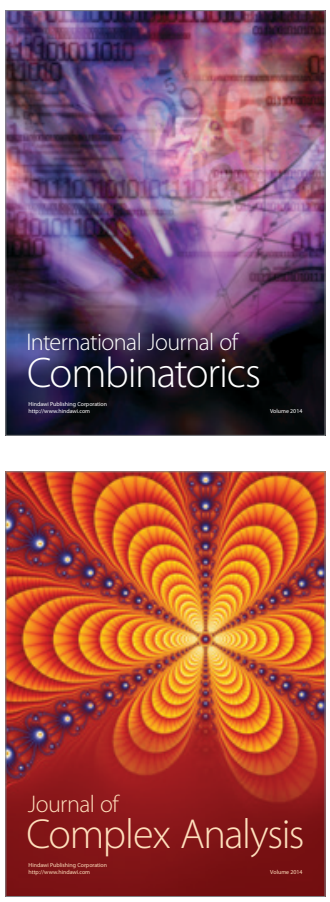

International Journal of

Mathematics and

Mathematical

Sciences
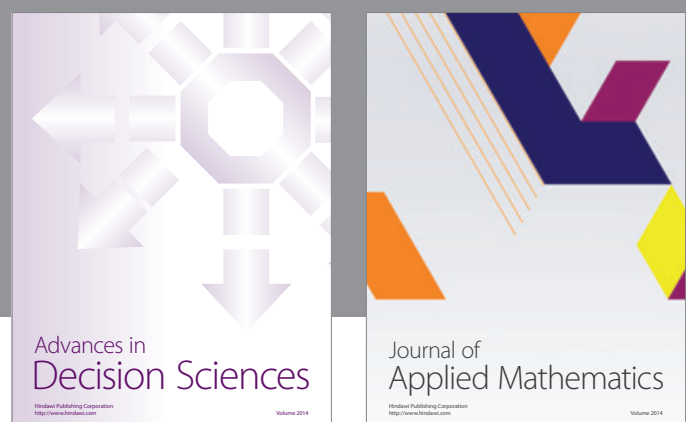

Journal of

Applied Mathematics
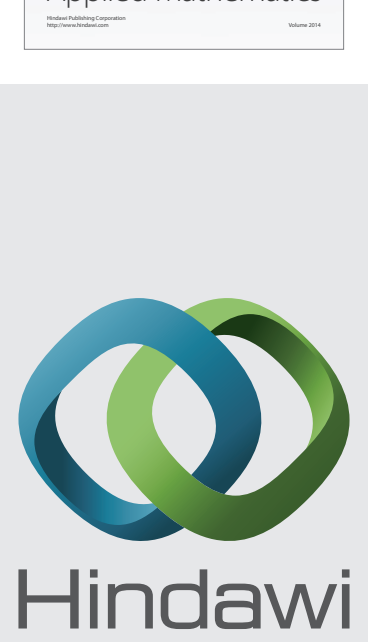

Submit your manuscripts at http://www.hindawi.com
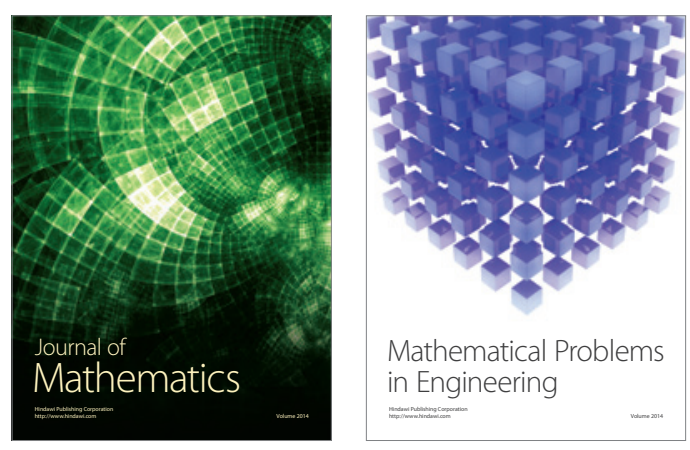

Mathematical Problems in Engineering


Journal of

Function Spaces
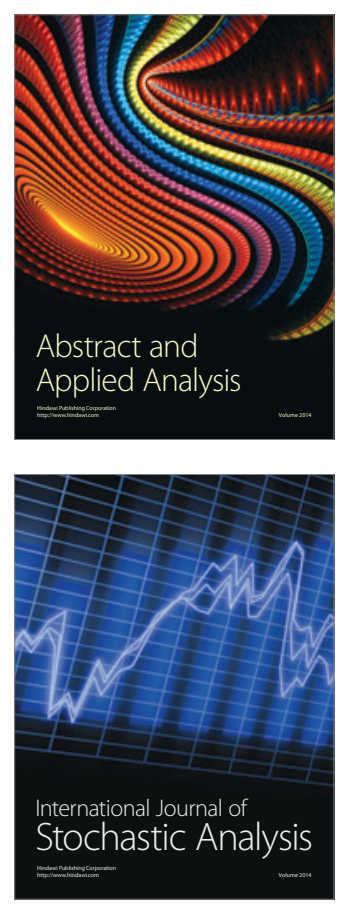

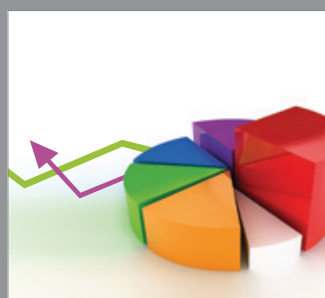

ournal of

Probability and Statistics

Promensencen
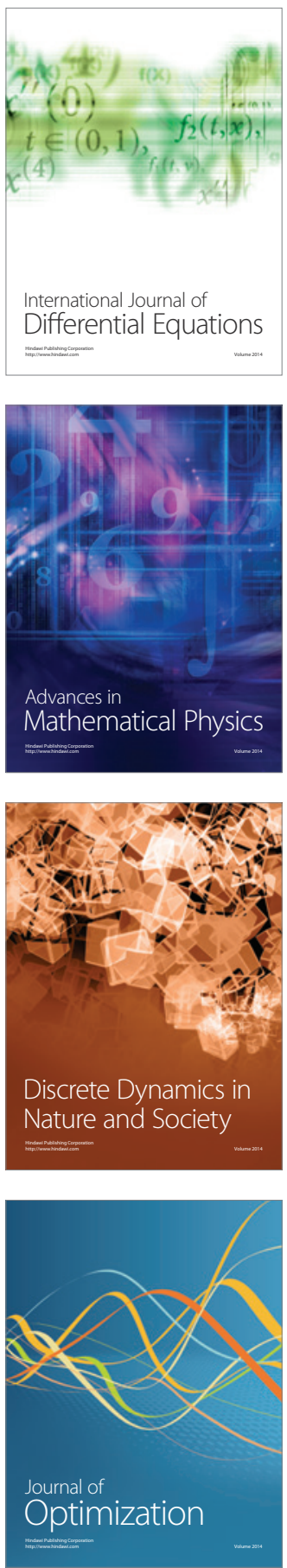